\title{
An evidence that SARS-Cov-2/COVID-19 spike protein (SP) damages hematopoietic stem/progenitor cells in the mechanism of pyroptosis in Nlrp3 inflammasome-dependent
} manner

\author{
Magdalena Kucia $\mathbb{D}^{1,2}{ }^{凶}$, Janina Ratajczak ${ }^{1}$, Kamila Bujko ${ }^{2}$, Mateusz Adamiak $\mathbb{D}^{2}$, Andrzej Ciechanowicz $\mathbb{D}^{2}$, Vira Chumak ${ }^{2}$, \\ Katarzyna Brzezniakiewicz-Janus ${ }^{3}$ and Mariusz Z. Ratajczak (iD) $1,2,3$ 瓜
}

(C) The Author(s), under exclusive licence to Springer Nature Limited 2021

Leukemia (2021) 35:3026-3029; https://doi.org/10.1038/s41375-021-01332-z

\section{TO THE EDITOR:}

Mounting evidence accumulates that hematopoietic stem/progenitor cells (HSPCs) and endothelial progenitor cells (EPCs) are damaged during severe SARS-Cov-2/COVID-19 infection $[1,2]$. It has been reported that patient infected with COVID-19 are frequently presented with anemia, lymphopenia, and thrombocytopenia [1-3]. This negative effect of the virus on human hematopoiesis and endothelium has been reported in infected patients and demonstrated in vitro after exposure of cells to SARS-Cov-2/COVID19 spike protein (SP) $[1,3,4]$. It is known that virus may enter cells and, directly in case of productive infection, lead to their irreversible damage. On the other hand, the interaction of viral SP with some of the receptors expressed on the cell surface may lead to their damage as well [1-3]. We have proposed that interaction of SP with the target cell surface receptors induces intracellular hyperactivation of Nlrp3 inflammasome which may lead to cell death by pyroptosis [5]. It is known that pyroptosis is characterized by the creation in a caspase-1 dependent manner of $\mathrm{N}$-gasdermin pores in the cell membrane, which leads to the release of cytosol components to extracellular space and final cell lysis [6].

As reported, SARS-CoV-2/COVID-19 enters human cells after binding to the angiotensin-converting enzyme 2 (ACE2) receptor utilizing SP for attachment and subsequent internalization. Moreover, transmembrane protease 2 (TMPRSS2) cleavage of SP may augment viral entry [7]. This facilitates its interaction with ACE2 and the subsequent fusion of viral and cellular membranes. The other receptor postulated to be involved in virus entry is toll-like receptor-4 (TLR4) [8]. Thus, this virus is using cell surface receptors that play a physiological role in the conversion of angiotensin II to angiotensin (1-7) (Ang [1-7]) as it is a case of ACE2 and TLR4 which belongs to pattern recognition cell surface receptor family and is responsible for inflammatory cytokine production and activation of the innate immunity responses. As demonstrated, both ACE2 and TLR4 are highly expressed on HSPCs and EPCs
$[3,4]$. In addition to these two receptors, SARS-CoV-2/COVID-19 may also interact with the extracellular matrix metalloproteinase inducer basigin, known as cluster of differentiation 147 (CD147) [9] as well as recently proposed with C-type lectin receptor and Tweety family member 2 [10].

In our recent perspective paper in Leukemia, we proposed that the adverse effects of infection on stem cell compartments result from the uncontrolled hyperactivation of the NIrp3 inflammasome [5]. To support this, we noticed that exposure of human UCBpurified $\mathrm{CD}_{34}{ }^{+} \mathrm{lin}^{-} \mathrm{CD} 45^{+} \mathrm{HSCs}$ to recombinant SP for $16 \mathrm{~h}$ lead to upregulation of mRNA expression for Nlrp3 inflammasome [4]. As determined by ELISA, we also detected elevated levels of IL-1 $\beta$ in the conditioned media (CM) from cells exposed to SP [4]. Release from cells of IL-1 $\beta$ in a caspase-1-dependent manner is an important indicator of Nlrp3 inflammasome activation.

This Letter to Editor reports that human $\mathrm{CD} 34^{+}$cells enriched for HSPCs and human $\mathrm{CD} 34^{+} \mathrm{CD} 133^{+} \mathrm{CD} 31^{+} \mathrm{CD} 144^{+}$cells enriched for EPCs activate Nlrp3 inflammasome after exposure to SP as evidenced by measuring the cytosolic activity of caspase- 1 by employing functional sensitive bioluminescent glow assay. Fig. 1A shows that caspase- 1 becomes activated in HSPCs in response to $\mathrm{SP}$, and Fig. 1B shows that the same phenomenon occurs in EPCs. Moreover, this activation has been inhibited after preincubation of SP with human recombinant ACE2 protein (rhACE2). Interestingly, activation of Nlrp3 inflammasome was higher in EPCs as compared to HSCs. Next, we exposed human $\mathrm{CD}_{3} 4^{+}$cells to SP in the absence or presence of Nlrp3 inflammasome-specific inhibitor MCC950 (Fig. 1C, D) and plated these cells in methylcellulose cultures to grow CFU-Mix and CFU-GM colonies. As demonstrated, exposure to SP decreased clonogeneic growth of CFU-Mix and CFU-GM, and inhibition of Nlrp3 inflammasome by MCC950 reversed this effect.

In the next set of experiments, we become interested in which receptors responsible for SP binding are accountable for the

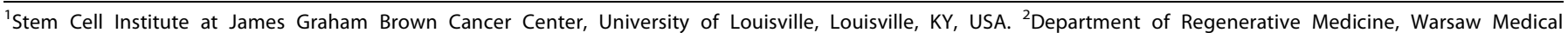

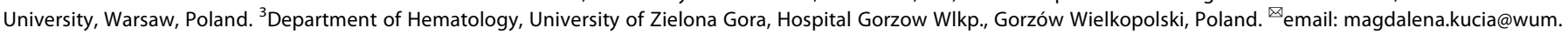
edu.pl; mzrata01@louisville.edu
}

Received: 6 May 2021 Revised: 10 June 2021 Accepted: 14 June 2021

Published online: 23 June 2021 

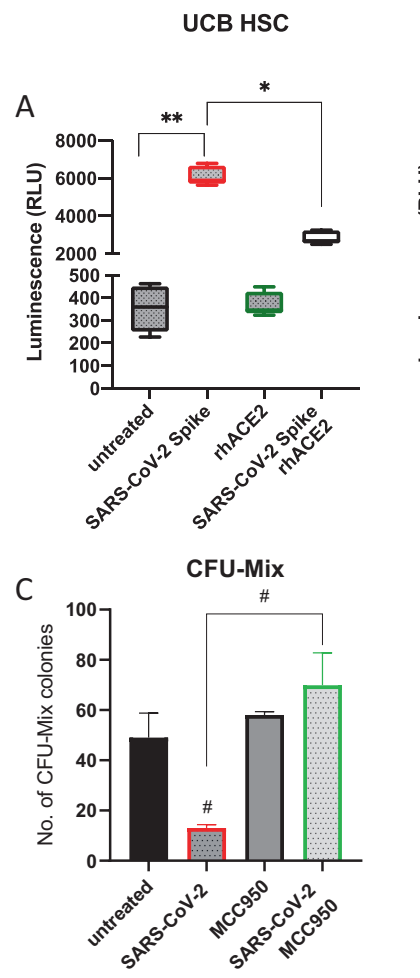
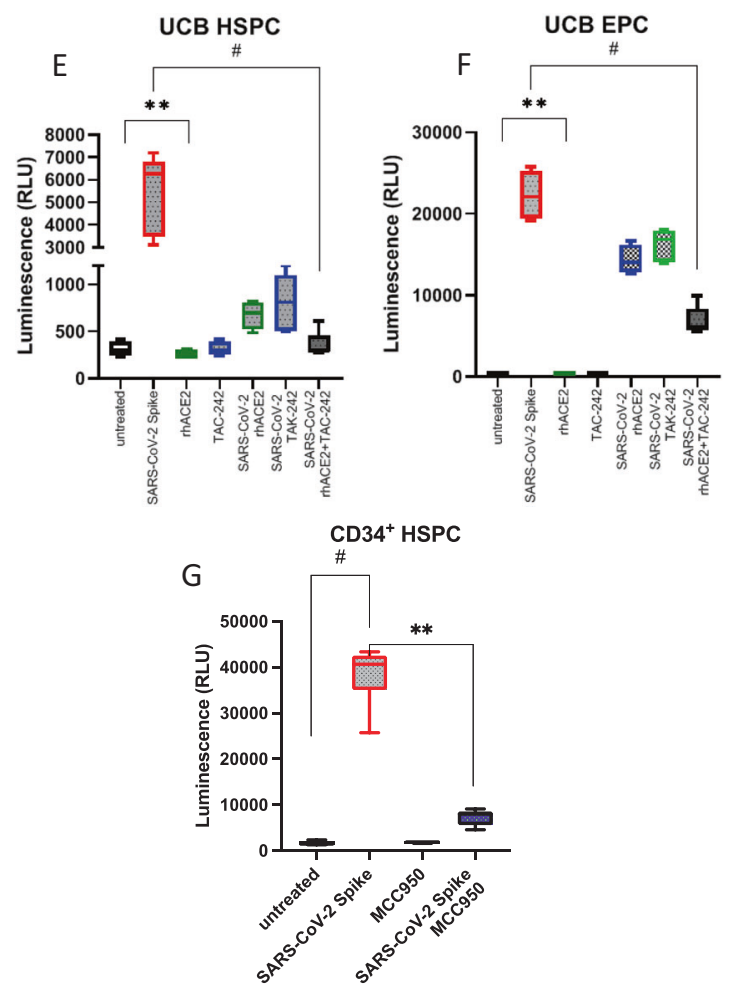

Fig. 1 Biological effects of COVID-19-derived SP on human HSPCs and EPCs. Effect of COVID-19-derived SP (20 nM, S1 + S2 ECD, His tag) and rhACE2 $(20 \mathrm{nM})$ after $16 \mathrm{~h}$ exposure of UCB-sorted HSCs (A) and EPCs (B) on activation of caspase-1 evaluated by Caspase-Glo 1 Inflammasome assay (Promega). Experiments were repeated three times ${ }^{*} p<0.01$ and ${ }^{* *} p<0.001$. C, D Nlrp3 inflammasome inhibitor MCC950 recovered clonogenic potential of UCB HSPCs from the inhibitory effect of COVID-19-derived SP. Cells were exposed to SP \pm MCC950 (10 $\mu M$ ). The data show the number of CFU-Mix (C) and CFU-GM (D) clonogenic progenitors obtained from UCB HSPCs. HSPCS were cultured in methylcellulose medium supplemented with appropriate cytokines, and CFU-Mix and CFU-GM colonies were counted using inverted microscope after 14 and 11 days of culturing. The data are presented as means \pm SEM. Experiments were repeated three times $\# p<0.01$. Effect of COVID-19-derived SP (S1 + S2 ECD, His tag) after $16 \mathrm{~h}$ exposure of UCB-sorted HSCS (E) and EPCs (F) on activation of caspase-1 evaluated by Caspase-Glo 1 Inflammasome assay (Promega). rhACE2 $(20 \mathrm{nM})$ and TLR4 blocking agent (TAK-242, $5 \mu \mathrm{M})$ we employed alone or together. Experiments were repeated three times ${ }^{* *} p<0.01$ and ${ }^{\#} p<0.0001$. G SP-mediated activation of Nlrp3 inflammasome in CD34 ${ }^{+}$HSPC was inhibited in a presence of MCC950 $(10 \mu \mathrm{M})$.

observed inhibition. We focused on SP interaction with ACE2 and TLR4 receptors (Fig. 1E, F). Therefore, in the set of similar assays to assess activation of Nlrp3 inflammasome by glow assay, we blocked the interaction of SP with ACE2 on the surface of cells by preincubation SP with rhACE2 and blocked TLR4 receptor on the surface of cells by employing specific TLR4 inhibitor TAC-242. Blockage of SP interaction with cells surface-expressed ACE2 and TL4 significantly inhibited activation of Nlrp3 inflammasome downstream mediator that is caspase-1. This inhibition was even more pronounced when the interaction of SP with both receptors was blocked simultaneously, however not completely inhibited, which suggests an involvement of other receptors in Nlrp3 inflammasome activation (CD147 or other toll-like receptor family members). Finally, Fig. 1G shows that SP-mediated activation of Nlrp3 inflammasome in $\mathrm{CD}_{3}{ }^{+} \mathrm{HSPC}$ was inhibited in a presence of MCC950.

Next, since as reported in an elegant paper that exposure of human HSPCs to SARS-CoV-2/COVID-19 derived recombinant SP inhibits the growth of hematopoietic clonogeneic progenitor cells [3], we performed clonogeneic assays for CFU-Mix, BFU-E, and CFU-GM where $\mathrm{CD} 34^{+}$cells before plating in methylcellulose were exposed to SP alone, or to SP in the presence of ACE2, TAC-242, or both ACE2 + TAC-242. As demonstrated in Fig. 2A, exposure of $\mathrm{CD}_{3} 4^{+}$cells to SP resulted in impairment of the clonogenic potential of these cells. Both numbers of colonies as well as their size decreased. At the same time, blockage of ACE2 interaction with ACE2 receptor and inhibition of TLR4 improved clonogenic growth of these cells. Finally, we exposed CD34 ${ }^{+}$cells to SP in the presence of a small molecular inhibitor of Nlrp3 inflammasome that is MCC950 and obtained similar results. Moreover, similar results we obtained with human EPC, in which colony formation was inhibited after exposure to SP (manuscript in preparation).

As mentioned above, hyperactivation of Nlrp3 inflammasome may induce cell death in the mechanism of pyroptosis that is characterized by the creation of gasdermin D pore channels in cell membranes, mediating the release in caspase-1-dependent manner of several biologically active danger-associated molecular pattern molecules [6]. One of the assays to detect pyroptosis is measuring lactate dehydrogenase (LDH) enzymatic activity in CM harvested from pyroptotic cells. Therefore, we measured the release of $\mathrm{LDH}$ in CM from human UCB CD34+ cells exposed to SP (Fig. 2B). Exposure to SP resulted in a significant increase in LDH level, which indicates the induction of pyroptosis in these cells. This effect was significantly reduced by adding rhACE2 and TAK242, as well as after exposure to MCC950. Thus, SARS-CoV-2/ COVID-19 may directly impair the viability and proliferative potential of HSPCs by hyperactivation of Nlrp3 inflammasome leading to pyroptosis. On the other hand, activation of Nlrp3 inflammasome in innate immunity cells may lead to cytokine storm and activation of the complement cascade and coagulation cascade affecting immune response against this pathogen [5].

To shed more light on molecular events responsible for SP effect on HSPCS, we have analyzed the proteome of HSPCsderived $\mathrm{CM}$ after exposure to recombinant SP (Fig. 2C). The 


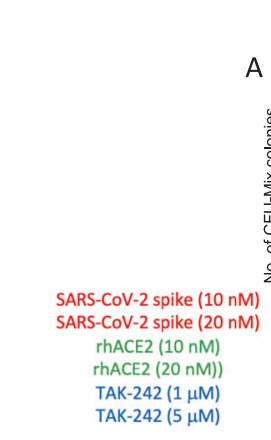

B

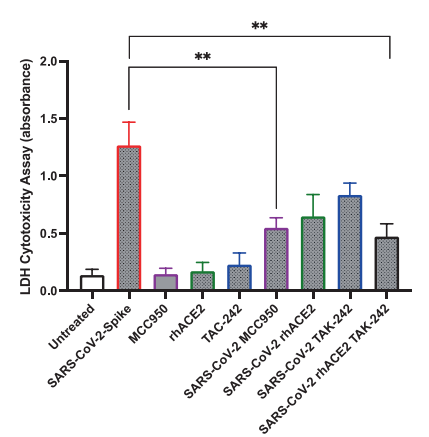

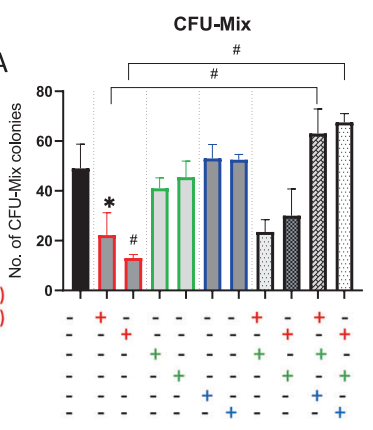

C

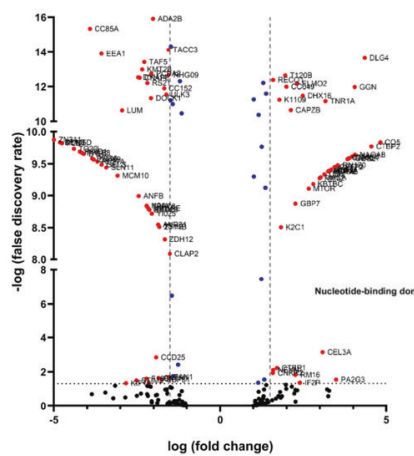

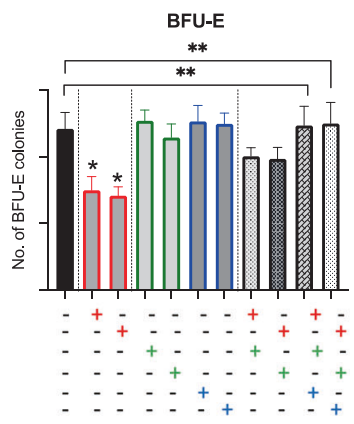

D

Fig. 2 Biological effects of COVID-19-derived SP on clononogenic growth and secretome of HSPCs. COVID-19-derived SP inhibits clonogeneic potential of human UCB CFU-Mix, CFU-GM, and BFU-E cells (A). Cells were exposed to SP \pm recombinant hACE2 or to SP \pm TLR4 inhibitor TAK-242. Experiments were repeated three times ${ }^{*} p<0.05,{ }^{* *} p<0.01$ and ${ }^{\#} p<0.005$. B After exposure to SP (10 nM), hACE2 (10 nM),

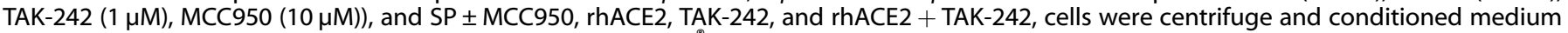
were harvested. LDH level was measured using CytoTox $96^{\circ}$ Non-Radioactive Cytotoxicity Assay (Promega) according to manufacturer protocol. Experiments were repeated two times ${ }^{* *} p<0.01$. C Volcano plot showing protein expression fold change values and their corresponding false discovery rate $(-\log 10 p$ value). Red points show statistically significant proteins with FDA $>1.3$. Volcano plot of condition medium proteome after spike stimulation of HSCs and normalized to the untreated group. Blue points show statistically significant proteins with fold change >1.5. D Graph showing the number of proteins found in the conditioned media analysis after stimulation of HSCs with Spike protein, Reactome.org annotated to the Immune system. The left side on the graph shows the individual signaling pathways involved in regulating the immune system to which the identified proteins are annotated. The categories of annotated proteins that are down-regulated are shown in blue, proteins that did not change theirs level in gray, and up-regulated proteins in red (Color figure online).

identified proteins with variable expression were then annotated to appropriate signaling pathways and biological processes using UniProt.org, Reactome.org, and KEGG databases (Fig. 2D). We have identified 441 proteins from which 311 were differently expressed after exposure to SP (142 proteins showed an increase in expression, and 169 proteins decreased expression). We observed that exposure to SP upregulates the expression of proteins involved in the positive stimulation of the immune system (C5, C7, PSMD4, K2C1, ICOSL, SH3K1, KPCB, CYFP2, and NTAL), TLR4 signaling pathway, and proteins that positively impact apoptosis, necrosis, and pyroptosis (mTOR, RBM10, and tumor necrosis factor receptor superfamily member $1 \mathrm{~A}$ ). Simultaneously, after SP exposure, we detected decrease in expression of proteins involved in the positive regulation of cell proliferation and differentiation (Fig. 2C).

In our current work, we focused on the interaction of SP with the two most important receptors binding SARS-Cov-2/COVID-19 on cells. We did not block other receptors, e.g., such as mentioned above CD147 or basigin that encodes the erythrocyte antigen Ok system. This receptor is expressed on erythroid cells and, as reported, serves as an essential receptor on red blood cells for the human malaria parasite, Plasmodium falciparum [11]. This may explain a current report showing direct infection of erythroid progenitors by SARS-Cov-2/COVID-19 leading to stress erythropoiesis [12]. Therefore, HSPCs at different specification levels could be affected differently by the virus depending on expression of viral entry receptors. We and others have reported that ACE2 and TLR4 are highly expressed on the most primitive subset of HSPCs $[3,4,13]$, and as demonstrated in this study, these cells are highly susceptible to SP. This study has been performed with the first available for research recombinant SP identified on SARS-Cov-2/ COVID-19. Currently, there are several variants of this SP, and the biological effects of these variants need further detailed studies [14]. Finally, all these results with SP have to be verified in similar experiments using pseudotyped and the best life variants of the virus.

What is also important and has to be addressed is if in the case of non-productive infection the virus can survive in a latent form after entry in the long-living HSCs. If this occurs, bone marrow and hematopoietic organs could be a reservoir of this virus. Further studies have also to assess the effect of inflammatory cytokines on the HSPCs compartment that are up-regulated during SARS-Cov2/COVID-19 infection and potential impact of activated proteolytic complement and coagulation cascades products. Finally, based on our data pointing to a pathological role of hyperactivated NIrp3 inflammasome in HSPCs, small inhibitors of this intracellular pattern recognition receptor may have a beneficial effect in ameliorating damage of HSPCs and EPCs during SARS-Cov-2/ COVID-19 infection [15]. This could be a therapeutic option in particular for the patients who are easy Nlrp3 inflammasome activators. Nevertheless, let us hope that the developed vaccines and current armamentarium of new drugs will lead to dethroning of this treacherous virus realm along with all of its relatives, with its stolen crown (corona). 


\section{REFERENCES}

1. Gupta A, Madhavan MV, Sehgal K, Nair N, Mahajan S, Sehrawat TS, et al. Extrapulmonary manifestations of COVID-19. Nat Med. 2020;26:1017-32.

2. Varga Z, Flammer AJ, Steiger $P$, Haberecker $M$, Andermatt $R$, Zinkernagel AS, et al. Endothelial cell infection and endotheliitis in COVID-19. Lancet. 2020;395:1417-8.

3. Ropa J, Cooper S, Capitano ML, Van't Hof W, Broxmeyer HE. Human hematopoietic stem, progenitor, and immune cells respond ex vivo to SARS-CoV-2 spike protein. Stem Cell Rev Rep. 2021;17:253-65.

4. Ratajczak MZ, Bujko K, Ciechanowicz A, Sielatycka K, Cymer M, Marlicz W, et al. SARS-CoV-2 entry receptor ACE2 is expressed on very small CD45(-) precursors of hematopoietic and endothelial cells and in response to virus spike protein activates the Nlrp3 inflammasome. Stem Cell Rev Rep. 2021;17:266-77.

5. Ratajczak MZ, Kucia M. SARS-CoV-2 infection and overactivation of Nlrp3 inflammasome as a trigger of cytokine "storm" and risk factor for damage of hematopoietic stem cells. Leukemia. 2020;34:1726-9.

6. Liu X, Zhang Z, Ruan J, Pan Y, Magupalli VG, Wu H, et al. Inflammasome-activated gasdermin $D$ causes pyroptosis by forming membrane pores. Nature. 2016;535:153-8.

7. Hoffmann M, Kleine-Weber $\mathrm{H}$, Schroeder S, Kruger N, Herrler T, Erichsen $\mathrm{S}$, et al. SARS-CoV-2 cell entry depends on ACE2 and TMPRSS2 and is blocked by a clinically proven protease inhibitor. Cell. 2020;181:271-80 e278.

8. Choudhury A, Mukherjee S. In silico studies on the comparative characterization of the interactions of SARS-CoV-2 spike glycoprotein with ACE-2 receptor homologs and human TLRs. J Med Virol. 2020;92:2105-13.

9. Wang K, Chen W, Zhang Z, Deng Y, Lian JQ, Du P, et al. CD147-spike protein is a novel route for SARS-CoV-2 infection to host cells. Signal Transduct Target Ther. 2020;5:283.

10. Lu Q, Liu J, Zhao S, Gomez Castro MF, Laurent-Rolle M, Dong J, et al. SARS-CoV-2 exacerbates proinflammatory responses in myeloid cells through C-type lectin receptors and Tweety family member 2 . Immunity. 2021. https://doi.org/10.1016/ j.immuni.2021.05.006.

11. Rowe JA, Opi DH, Williams TN. Blood groups and malaria: fresh insights into pathogenesis and identification of targets for intervention. Curr Opin Hematol. 2009;16:480-7.
12. Huerga Encabo H, Grey W, Garcia-Albornoz M, Wood H, Ulferts R, Aramburu IV. et al. Human erythroid progenitors are directly infected by SARS-CoV-2: implications for emerging erythropoiesis in severe COVID-19 patients. Stem Cell Rep.2021;16:428-36.

13. Ropa J, Trinh T, Aljoufi A, Broxmeyer HE. Consequences of coronavirus infections for primitive and mature hematopoietic cells: new insights and why it matters. Curr Opin Hematol. 2021;28:231-42.

14. Huang Y, Yang C, Xu XF, Xu W, Liu SW. Structural and functional properties of SARS-CoV-2 spike protein: potential antivirus drug development for COVID-19. Acta Pharm Sin. 2020;41:1141-9.

15. Ratajczak MZ, Kucia M. The Nlrp3 inflammasome-the evolving story of its positive and negative effects on hematopoiesis. Curr Opin Hematol. 2021. https:// doi.org/10.1097/MOH.0000000000000658.

\section{ACKNOWLEDGEMENTS}

This work was supported by NIH grants 2 R01 DK074720 and the Polish National Center OPUS grants UMO-2018/29/B/NZ4/01470 to MZR.

\section{COMPETING INTERESTS}

The authors declare no competing interests.

\section{ADDITIONAL INFORMATION}

Correspondence and requests for materials should be addressed to M.K. or M.Z.R.

Reprints and permission information is available at http://www.nature.com/ reprints

Publisher's note Springer Nature remains neutral with regard to jurisdictional claims in published maps and institutional affiliations. 